\title{
Is there a right to get a right diagnosis whenever possible? Ethical and social issues in dealing with undiagnosed rare diseases
}

Sabina Gainotti, Bioethics Unit, Office of the President, Istituto Superiore di Sanità, Rome, Italy. Sabina.gainotti@iss.it

Deborah Mascalzoni, University of Uppsala, Uppsala, Sweden. Deborah.mascalzoni@crb.uu.se

Virginie Bros Facer, EURORDIS, Paris, France. virginie.bros-facer@eurordis.org

Carlo Petrini, Bioethics Unit, Office of the President, Istituto Superiore di Sanità, Rome, Italy.

Carlo.petrini@iss.it

Giovanna Floridia, Bioethics Unit, Office of the President, Istituto Superiore di Sanità, Rome, Italy. Giovanna.floridia@iss.it

Marco Roos, Leiden University Medical Centre, Leiden, Netherlands. M.roos@lumc.nl

Marco Salvatore, National center for rare Diseases, Istituto Superiore di Sanità, Rome, Italy.

Marco.salvatore@iss.it

Domenica Taruscio, National center for rare Diseases, Istituto Superiore di Sanità, Rome, Italy.

Domenica.taruscio@iss.it

Corresponding author: Sabina Gainotti 


\section{Abstract}

The time required to reach a correct diagnosis is one of the most important problems for rare disease (RD) patients.

Diagnostic delay can be intolerably long, to the point that it is usually described as a "diagnostic odyssey" and, sometimes, a diagnosis might never occur.

The International Rare Disease Research Consortium proposed, as ultimate goal for 2017-2027, to enable all people with a suspected RD to be diagnosed within one year if the disorder is known, and to enter a globally coordinated diagnostic and research pipeline for the unsolved cases.

In-depth analysis of the genotype through next generation sequencing, together with a standardized indepth phenotype description and sophisticated high-throughput approaches, have been applied as diagnostic tools to increase the chance of a timely and accurate diagnosis.

This approach is very fruitful as, according to the Orphanet database, from 2010 to March 2017 more than 600 new RDs have been described and about 3600 genes linked to RDs have been identified.

However, combination of -omics and phenotype data and international sharing of this information raise ethical concerns. Values to be assessed include not only patient autonomy but also family implications, beneficence, non-maleficence, justice, solidarity and reciprocity, which must be respected and promoted and, at the same time, balanced among each other.

In this work we suggest that, to maximise patients involvement in the search for a diagnosis and identification of new causative genes, undiagnosed patients should have the possibility to:

1) actively participate in the description of their phenotype; 2 ) choose the level of visibility of their profile in matchmaking databases; 3 ) express their preferences regarding return of new findings, in particular which level of Variant of Unknown Significance (VUS) significance should be considered relevant to them.

The quality of the relation between individual patients and physicians, and between the patient community and the scientific community is critically important for making the best use of available data and combining efforts in the search for matches with similar cases worldwide that will help to solve unsolved cases.

The contribution of patients to collecting and coding data comprehensively is critical for efficient use of data downstream of data collection.

\section{Keywords}

Undiagnosed rare diseases; diagnostic odyssey; NGS; deep phenotyping; genomic matchmaking; secondary findings; patient involvement; 


\section{Background}

Making a diagnosis for a rare genetic disorder can be very challenging. The known number of rare genetic diseases (RDs) is currently 6000-8000 [1] with 250-280 new diseases described annually [2], and the most rare are estimated to affect about $1 / 2,000,000$ patients [3].

The time required to reach a correct diagnosis is one of the most important problems for rare disease (RD) patients and, sometimes, a definitive diagnosis might never occur.

As outlined within the "International recommendations to address specific needs of undiagnosed rare disease patients" there are two different groups of undiagnosed patients [4]:

- 'Not yet diagnosed' refers to patients suffering from known conditions that should be diagnosed but haven't been because they have not been referred to the appropriate clinician or clinical centre;

- Undiagnosed ("Syndromes Without A Name" or SWAN) refers to patients for whom a diagnostic test is not yet available since their diseases have not been characterised and the cause(s) is not yet identified.

In both cases, similar measures can be applied to increase the chance of getting a timely and accurate diagnosis and they relate to the organisation of healthcare services, which must include interdisciplinary teams of well trained professionals, and to the use of available diagnostic tools and platforms for the sharing of clinical and genetics data.

According to Nick Black et al. the "diagnostic odyssey" of RD patients encompasses three different periods: patient interval; primary care interval; and specialist care interval [5].

The patient interval goes from the "onset of symptoms and/or signs", i.e. when the patient or parent notices for the first time what will later be classified as a symptom or sign of the disease, to the "presentation to care", when the patient/parent is seen for the first time in primary care. The primary care interval goes from "presentation to care" to "referral to specialist care", when responsibility for the diagnosis shifts from primary physician to specialist care who will hopefully result in receiving an accurate diagnosis.

The length of patient interval may depend on the frequency and seriousness with which signs and symptoms present and on the patient and parents' capacity to recognise them. While a sign is an objective evidence of a disease (such as a skin rash or a cough), and can be detected by someone different from the individual affected, a symptom is a phenomenon that is experienced (such as stomach ache, lower back 
pain, and fatigue) and can only be recognized by the individual who is experiencing it. Being subjective, symptoms may not be timely recognised.

In both primary and specialised care, diagnostic delays and/or errors may occur for several reasons.

Clinical diagnosis is a medical act based on information coming from many sources such as findings (signs and symptoms) from a physical and/or psychological examination of the patient, interview with the patient or family or both, medical history of the patient and family, clinical findings as reported by laboratory and instrumental tests.

In the diagnostic interview, which plays a vital role in the diagnostic process, the physician typically tends to consider complex and rare diseases only after discarding the most common and simple ones [6].

Besides, the physician may either not know the specific manifestations of the disease or may not have performed the necessary and appropriate diagnostics tests.

The patient may present atypical symptoms and/or clinical manifestations for a known disorder, or a combination of symptoms for more than one disorder, or even a novel, unreported disorder.

There may be instances in which a non-genetic risk factor is implicated, but not clearly identified, like a rare syndrome associated with the use of a certain drug or with the exposition to multifactorial environmental factors [7].

In addition, there may be communication problems between patient and physician, especially when the patient is a minor who is not able to speak, due to the young age or to disabilities and difficulties to express him/herself, and when common symptoms, like fatigue or pain, do not appear to have an obvious medical cause.

Even though some delay is to be expected in the diagnosis of a rare disorder, this delay can be intolerably long.

In a survey carried out by EURORDIS on eight relatively "common" RDs (Crohn's disease, Cystic fibrosis, Duchenne muscular dystrophy, Ehlers-Danlos syndrome, Marfan syndrome, Prader Willi syndrome, Tuberous sclerosis, Fragile $X$ syndrome), 25\% of patients reported to have waited from 5 up to 30 years to get the right diagnosis, the initial diagnosis being wrong in $40 \%$ of cases [8].

Although exact statistics are not available, the situation is likely worse for the rarest disorders.

The impact of such experience is immense for the single patient and his/her family, and costly to society. 
Diagnostic delay prevents doctors from initiating the right therapeutic regimen when one exists, and it involves useless visits and exams, some of which are invasive and painful, resulting in an increased stress for the patient and increased costs for the healthcare services.

There are psychological and social consequences related to the uncertainty of this special situation. Not recognizing a patient's symptoms by physicians, patient's family members, friends, colleagues and/or others can lead to misunderstandings in a patient's social environment, a sense of isolation and even stigmatisation of the patient or his/her parents.

Parents of a child with an undiagnosed disorder are concerned that their other children might be similarly affected. There are also implications for reproductive choices as the absence of diagnosis prevents families from making informed decisions [9].

Diagnostic delay prevents patients from accessing specialised healthcare and social services, such as periodical follow up that are standard practice for other severe and chronic conditions (i.e. for cancers).

Access to care is linked to integrated clinical paths that are tied to specific codified diagnoses. Thus, faulting clear diagnosis patients may be denied access to the care or may be forced to rely upon their own finances and resources.

A delayed or missed diagnosis may create undue inequalities in comparison with other categories of patients that can access diagnosis more readily. Therefore, we may suggest that, whenever possible, there is a duty to pursue a timely and accurate diagnosis, or at least guarantee access to care for patients where clinical observations are inconclusive with respect to a specific codified diagnosis. As we discuss later, this is important for the development and application of innovative diagnostic methods and automated decision support towards mitigating rare symptoms.

Several international initiatives have been developed to approach the problem of undiagnosed diseases.

In 2008, the NIH Undiagnosed Disease Program (UDP), through the efforts of the National Human Genome Research Institute, the NIH Clinical Center, the Office of Rare Diseases Research, and other NIH research institutes and centers, arose to provide a diagnosis for individuals who had long sought one without success [10].

Starting from the US experience and prompted by parents of undiagnosed children, the Undiagnosed Diseases Network International (UDNI) was established in 2015 and it currently includes Australia, Canada, Japan, Israel, India, Japan, Korea, Sri Lanka, Thailand, United States and 9 European Countries (Austria, 
Belgium, France, Germany, Hungary, Italy, Spain, Sweden, The Netherlands) together with patient organisations. The UDNI Members share the strategy, general principles and best practices to tackle undiagnosed patients, with respect to Country specificities [11, 12].

Besides concentrating expertise and capacities in medical research centres dedicated specifically to undiagnosed cases, the diagnostic tools that are currently implemented to increase the chance of getting a timely and accurate diagnosis consist in the in-depth analysis of a patient's genotype through the use of next generation sequencing (NGS) techniques, coupled with a standardised in-depth phenotype description $[13,14]$.

The increasing ability to combine multiple types of data provides new possibilities for finding diagnostic markers or patterns. For instance, it is possible to combine sophisticated high-throughput approaches, such as RNA sequencing and -omics technologies, with functional studies to validate the pathogenicity of variants [15].

When the case remains unsolved, it is still possible to search for other patients with similar genotype and phenotype features worldwide by using matchmaking platforms, in order to narrow the number of candidate genes that are associated with a particular phenotype [16].

The current approach to diagnostic research on RDs described above is proving to be very fruitful as, according to the Orphanet database, since 2010 up to March 2017 more than 600 new RDs have been described and about 3600 genes have been identified as linked to RDs [17].

On this note the "International Rare Diseases Research Consortium" (IRDiRC)'s 2020 goal for 200 new therapies was achieved in early 2017, 3 years ahead of schedule, and has recently published its next ambitious goal for 2017-2027 that is to enable all people coming to medical attention with a suspected RD to be diagnosed within one year, if the disorder is known in the medical literature, and to propose patients with unknown disorders to enter a globally coordinated diagnostic and research pipeline [18].

However, like other scientific innovations and methodological approaches which carry new potential benefits and foreseen or unforeseen risks, the combination of -omics and phenotype data and international sharing of this information raise ethical concerns that need to be addressed. Values to take into account in the assessment include and are not limited to patient autonomy as well as family implications, beneficence, non-maleficence, justice, solidarity and reciprocity (Table 1) which must be respected and promoted and, at the same time, balanced among each other. 


\section{Getting accurate diagnosis through integrated genotype and phenotype analysis}

In the medical literature, the term "diagnosis" can have different meanings. A "clinical diagnosis" refers to the integrated information of findings detected by a clinician after physical examination of the patient, interview with the patient or family or both, medical history of the patient and family, and clinical findings as reported by laboratory and instrumental tests. A histological diagnosis is the microscopic description of tissues and cells; genetic diagnosis describes a genetic mutation, regardless of whether the gene function is known.

The emerging era of "Precision medicine" is providing a new and additional dimension to the concept of diagnosis.

Precision medicine has been defined as "treatments targeted to the needs of individual patients on the basis of genetic, biomarker, phenotypic, or psychosocial characteristics that distinguish a given patient from other patients with similar clinical presentations"[19].

According to William Gahl, coordinator of the NIH Undiagnosed Diseases Program (UDP), the definition of diagnosis that is most satisfying for the aims of precision medicine includes an understanding of disease pathogenesis, linking genetic and clinical findings and informing prognosis and therapy [13].

With regard to genotyping, Next Generation Sequencing (NGS) is increasingly being applied in clinical diagnosis, as it can identify genetic variations associated with disease, can determine fusion genes and can detect pathogens on patient samples. Unlike previous diagnostic sequencing, NGS can deliver a full qualitative and quantitative analysis of the DNA or RNA sequences within a sample in a single test and thereby it holds the promise of improved diagnosis; moreover, the use of these advanced methodologies has accelerated the pace of discovery by enabling hypothesis-free approaches $[20,21]$.

However, the application and analysis of NGS data and the interpretation of variants of unknown clinical significance (VUS) remain a major challenge in diagnostics, particularly as resources for functional studies enabling pathogenic validation are limited. The development of high-throughput models of disease that use patient-derived material might help to clarify the implications of VUS. Furthermore, sharing of data on VUS among institutes is essential to identify overlapping results and determine the pathogenic significance of these variants.

Reference standards are an invaluable resource through which to understand these limitations [22]. Also, the complexity of data processing, analysis and interpretation requires the development of appropriate 
bioinformatics tools combining the results of sequencing data with in-depth phenotype data with trained and qualified experts competent in the complex analysis of the data.

It has been reported that in highly detailed phenotyped RD cohorts, the diagnostic yield of NGS approaches $40 \%$, while with less specific phenotypes, the rate of diagnostic success is far smaller [23].

Deep phenotyping has been defined as "the precise and comprehensive analysis of phenotypic abnormalities in which the individual components of the phenotype are observed and described, often for the purposes of scientific examination of human disease" [24, 25].

In order to be suitable for integrated analysis with genotype data, phenotype data need to be collected according to standardized terminologies and ontologies, often with annotations and links to other information [26].

There are several ontologies that are currently being applied to the study of human diseases [27, 28].

Most projects on undiagnosed RDs (e.g., the US National Institutes of Health Undiagnosed Diseases Program, Care4Rare Canada and Australia, EURenOmics, NEUrOmics, RD-Connect, the International Standards for Cytogenomic Arrays Consortium, national or regional undiagnosed disease programmes) are implementing and contributing to the development of the Human Phenotype Ontology (HPO), a comprehensive hierarchy of approximately 11,000 standard terms, most of them with detailed definitions and alternative names $[29,30]$.

The HPO itself does not describe individual disease entities but, rather, the phenotypic abnormalities associated with them, which may be shared between other RDs and common diseases as well.

Currently there are 21 research projects and 25 tools using HPO terms, some of which (i.e. the software Phenotips [31] and Phenomizer [32]) were developed to be used by clinicians, to assist them in the diagnosis of patients.

The Monarch Initiative, a collaborative, open science effort that aims to integrate genotype-phenotype data from many species and sources in order to support precision medicine, developed Guidelines on "How to annotate a patient's phenotypic profile" [33].

The Guidelines recommend that the physician reports "negative annotations" while describing a patient phenotype, which are the traits that were expected to be found within a defined clinical framework, but were actually not found.

Some matchmaking platforms also allow the clinician to indicate the severity of observed phenotypes [34]. 
When, despite all efforts, the case remains unsolved or SWAN, there are several data sharing and matchmaking platforms at the international level that have been developed in order to find matches between similar cases.

Matchmaking platforms are being developed by Universities, medical centres, and research institutions that are creating repositories of undiagnosed cases through the collection and comparison of genotypic and phenotypic data based on the HPO or other ontologies [35, 36, 37].

These initiatives are joining their efforts in order to allow the communication of specific case details within larger shared environments.

Matchmaker Exchange (http://www.matchmakerexchange.org/) is a project supported by the Global Alliance for Genomics and Health (http://genomicsandhealth.org/), the International Rare Disease Research Consortium (http://www.irdirc.org/), and ClinGen (http://clinicalgenome.org/) and represents the largest effort in this regard [38]. Matchmaker Exchange can also serve as a paradigm for a wide range of pattern matching services, i.e. beyond genetic mutations and phenotypes only, when generalizing its requirement for standardized data. Many patient or disease data collections contain substantial amounts of data that cannot be directly classified as human phenotypes. Their standardization, for instance as part of a FAIRification procedure $[39,40]$, will create a wide range of options for discovering diagnostic markers.

\section{On the appropriateness of prescribing NGS techniques for undiagnosed cases}

The great potential of NGS techniques are generating new hopes among undiagnosed patients. Indeed, to the point that SWAN Europe, the European coalition of associations specifically dedicated to undiagnosed patients lists "facilitating access to genomic technologies for families within the undiagnosed community" among its main objectives [5].

The optimal timing for Whole Exome Sequencing (WES) in the diagnostic process is still unclear, whether it should be at the first medical encounter after a clinical evaluation where a significant genetic heterogeneity is evident, or after having performed first tier of gene tests, or towards the end of the diagnostic odyssey when extensive and possibly invasive tests have already occurred [41]. Indeed, the indication for WES becomes clear once no pathogenic variant is detected in the first five candidate genes in the clinical suspicion list. However, it's noteworthy to underline the frequent waiting time between each individual test result and the cost to the patient (both financially and emotionally) for each recurrent clinic visit. 
NGS, and in particular WES, has proved to have a high diagnostic utility and to be cost effective in undiagnosed patients as it allows to dramatically reduce both the number of tests and the time required to get an accurate diagnosis, thus reducing the financial and psychological burdens associated with prolonged investigation $[42,43,44,45,46]$.

However, the current bottleneck is the waiting list not because of issues related to the technologies but because of shortage of qualified experts for the analysis of the sequencing results. Therefore, there is a huge need to increase capabilities and training of experts to analyse these findings. Currently this availability may present great variations from country to country. Moreover, even though the costs of NGS tests are falling dramatically, with the expected increasing use of genome and exome sequencing, the costs of storage of these huge data might become a critical issue.

Based on the UK number and extrapolating to the European population, SWAN Europe have estimated that more than 65,000 children are born in Europe each year with a syndrome without a name [47].

It is likely that by summing up the number of "yet to be diagnosed" and "SWAN" patients, the request to perform NGS techniques in all patients who are lacking accurate diagnosis will become evident to healthcare systems and competent authorities in the near future.

Thus, questions remain regarding which undiagnosed patients should be prioritised for NGS analysis, who should interpret the results by what criteria and who should return results, and which resources are available or need to be developed to this aim. This may raise the question in which steps medical doctors (MD) need to be involved, because this issue typically introduces a bottleneck. It appears reasonable to envision a situation where experts trained in modern data interpretation aid MDs and patients, thus reducing the demand on MDs in the overall process.

A model to take into consideration is the NIH UDP for examples as well as others, where the decision is taken on a case-by-case basis with the involvement of multiple RD experts, largely through consultations during UDP admissions. The program has been able to achieve half of the diagnosis through massive parallel sequencing from phenotype informed genetic testing, the other half through biochemical and radiologic investigations and molecular analyses $[48,49]$.

This model indeed is being used by other UDP programs worldwide $[11,50]$ and modified taking into account the specific context in each country. Despite this, the US UDP model has also been criticised for being successful in only a small proportion of the patients evaluated, for not necessarily resulting in treatment outcomes, and for being funded by the NIH "without any consideration of cost" [51]. 


\section{Improving diagnostic and discovery processes through patient involvement}

Undiagnosed patients are obviously highly motivated to participate in the diagnostic process by searching information on specific variants in the scientific literature and undertaking individual initiatives throughout social media to spread information on their particular genotype and phenotype characteristics and search for individual cases still unpublished [52].

They are also likely to participate in biobank and registry based research, in matchmaking databases [53, $54,55]$ and they are actively contributing to starting dedicated programs for undiagnosed diseases, as in the experience of the UDNI [11].

The search on dedicated platforms for other patients worldwide, sharing rare genotype and phenotype features has relevant ethical implications for undiagnosed patients, in particular relating to respect for their autonomy, protection of confidentiality and communication of new findings as they emerge, and involvement of children in research.

Here we propose that, in order to maximise patient involvement in the search for a diagnosis and in the identification of new causative genes, undiagnosed patients should be offered the possibility to actively participate to the diagnostic process (box 1):

As regard to the involvement of children we propose that, as long as they are intellectually able to make these decisions, they must be involved as soon as possible in the decision making to determine whether to share or not their data, with whom especially if data include pictures and are easily searchable by their peers.

The first request, patient participation to phenotype description, is motivated by the possibility that clinicians and patients consider different phenotypic traits as more or less relevant in a patient's phenotype, especially when a patient is suffering for "medically unexplained symptoms" [56].

The concepts of "illness' and" "disease" describe the different perspectives from which patients and doctors may look at the same phenomenon. "Illness" stands for the ill health as it is experienced by the patient, while "disease" stands for the disease as it is diagnosed by the physician following the results of medical examinations, tests and investigations, according to standardised diagnostic codes [57].

In the translation from "illness" to "disease", a practitioner may filter the information provided by the patient according to the relevance $s /$ he attributes to certain signs or symptoms for their coherence with predefined hypothesis. 
The role of a physician's hypothesis is indeed fundamental in the diagnostic process, since it provides a framework to organise the information gathered and to actively search for additional information.

However, a physician might dismiss phenotypic features that are not coherent with the main hypothesis but are relevant to the patient. Therefore, the patient should be entitled to review his/her phenotype description and eventually notify the relevant physician if $s / h e$ feels that relevant phenotypic traits are missing or different from what is reported.

To encourage patient involvement, the Monarch Initiative has translated phenotype descriptions into plain language without clinical terms unfamiliar to patients [58]. It has already developed a layer of 5,000 corresponding terms understandable for patients, basic research scientists, clinicians, and machines alike to help improve disease characterization and diagnosis.

Patient involvement enables the scientific community to extend ontologies, including HPO terms, with more precise terms, including for instance more subtle auditory, visual, and sensory traits identified by the patient community [55].

As regard to the second request, the visibility level of the profile in matchmaking databases, patient involvement is equally important in the decision regarding his/her privacy settings: there are different matchmaking databases for unsolved rare diseases that use automated matching systems to connect users who are contributing data with strong genotypic and phenotypic similarities.

Regulations focus on security of handling data (IT security measures) and on anonymity. However, this may not be the primary concern for RD patients. While protecting confidentiality and applying all the possible measures to ensure proper access is still critical [59], other preoccupations may be more important in the search for a diagnosis [60].

For the purposes of increasing the number of matches that are currently possible by using existing platforms, it would be ideal if the described cases were routinely made available to a wide albeit closed community of researchers if not completely public, in accordance with national privacy regulations and with the preferences of the patient.

For a number of reasons, including the wish to protect the patient's privacy or the wish to be the first to identify and publish the causative or implicated gene(s), a researcher may decide to keep the patient record "private" or to share it with a limited number of researchers thus limiting the possibility to find matches in wider matchmaking networks. 
While a researcher can be aware of the risks deriving from wider data sharing, the discussion should always be pursued with the patient.

Acknowledging a patient's will may really provide a balance between unnecessary paternalism and undue liberalism in dealing with undiagnosed patients' data.

An IRDiRC / Global Alliance for Genomics and Health (GA4GH) working group is currently investigating privacy-preserving record linking to overcome the problem of patient identification while still enabling data from the same patient in multiple data sources to be combined [61].

The third request is that undiagnosed patients should be offered appropriate genetic counselling in order to be involved in deciding how much significant must a variant be in order to be communicated as a "secondary finding".

According to the American Society of Human Genetics genetic counselling is a "communication process which involves an attempt by one or more qualified persons to help the individual or family to: 1 ) comprehend the medical facts, including the diagnosis, probable course of the disorder, and the available management; 2 ) appreciate the way heredity contributes to the disorder $(. .) ; 3$.$) understand the$ alternatives for dealing with the risk of occurrence; 4) choose the course of action which seems to them appropriate $(\ldots) ; 5$.$) make the best possible adjustment to the disorder (. .$.$) and/or the risk of recurrence of$ that disorder" [62].

With the use of NGS techniques, the likelihood to identify variants that are not directly linked with the known/expressed phenotype is high. While diagnostic variants rely on the fact that patients are aware and looking for that specific answer, secondary variants may be revealed unexpectedly carrying unwanted implications that may add to an already delicate situation. At the same time providing adequate information in the consent process may be a challenge since counselling cannot be provided beforehand on too many variants.

To deal with this complexity, models of multistage consent have been proposed for the return of findings $[63,64]$. These models foresee that patients can provide a general answer for being re-contacted and delay the final decision until more information is available for better informing the decisional process.

Along with the consent models, another decisional process includes deciding which variants should be provided to patients. It has been suggested that results originating from sequencing (whether related to the original research question or not) that are "actionable" as they have acceptable clinical validity and important impact on patients' health should be returned to participants (for example BRCA 1 or BRCA2 
mutations) [67]. However, the threshold for "acceptable" clinical validity and impact are a moving target and are difficult to define and there is a good deal of disagreement among experts. This considerations apply also to the "actionability" concept that falls among the criteria for returning results. In fact, a result can be considered to have clinical utility when actions can be taken on its basis and, according to different interpretations, this may be limited to therapeutic and preventive measures or it may include reproductive choices and other decisions that are relevant for the patient. In addition, it must be remembered that clinical utility has different meanings in different health care contexts, in which different preventive or therapeutic interventions can be offered.

For an undiagnosed patient, other priority criteria may apply: the possibility to actively search information on the new variant and eventually contact other carriers to organize common actions in order to raise attention of the scientific community on the variant at stake makes the information equally actionable.

Pioneering projects and programs dealing with undiagnosed diseases have reported their experiences regarding returning results to participants. For example, one study carried by the Canadian FORGE project using focus groups, revealed that, in general, parents want to receive as much information about their child's health as possible as they perceive that the benefits of receiving all incidental findings outweigh the potential harm [66].

Thus, undiagnosed patients are usually willing to be informed about new findings, independently from the immediate clinical utility. A cost benefit ratio still needs to be applied. But for those results that are not related to the RD under study, in fact constitute a completely different category that may be looked at given that the entire sequence is produced anyway. This is a form of opportunistic screening where, as the term suggests, screening for other genes is conducted because it is convenient (and supposedly useful) [67]. Regarding these results, RD patients should hold the same right to access results as the general population $[68,69]$.

\section{Discussion}

One of the main goals for the RD community, as defined by the IRDIRC, is to deliver the means to diagnose most RDs, including identification of all RD-associated genes.

Recently, the IRDIRC proposed as its ultimate goal for 2017-2027 to enable all people coming to medical attention with a suspected RD to be diagnosed within one year if the disorder is known, and to propose all currently unsolved cases to enter a globally coordinated diagnostic and research pipeline [18]. 
To achieve this, the scientific and the patient community need to work jointly to implement organizational solutions that make the best use of available technologies by pooling knowledge and expertise, registries, data and funding. In the EU this is happening thanks to the establishment of 24 European Reference Networks (ERN) for research and care of rare and complex diseases, where experts are able to share cases through virtual meetings and review outcomes retrospectively to agree on the best possible care [70].

Hopefully the availability of new tools, such as NGS and deep standardised phenotyping, enable physicians, researchers, and patients to find matches with data of other patients and discover patterns that can lead to appropriate and targeted diagnostic tests.

Besides, the current trend to standardise phenotype description and other types of observations by using ontologies, carries the potential for a more thorough comprehension and mutual understanding between patients and clinicians who can both contribute in building and applying a common vocabulary. Importantly, unambiguous coding of data in terms of machine readable ontologies opens new gateways for computational support.

However, there may still be ethical, legal constraints and socio cultural barriers to data sharing and matchmaking that limit the capacity to fully exploit the potential of existing tools and the size of matchmaking networks, thus limiting the potential to identify matches.

According to Boycott et al, it takes approximately 2-3 years to identify an additional unrelated individual with a pathogenic mutation in the same gene after publication of a single patient or family illustrating the difficulty in identifying unrelated patients with similar phenotype and pathogenic variants in the same gene. The number of candidate genes (e.g. containing deleterious-appearing genetic variation) that are unpublished and/or in inaccessible "silos" worldwide, is estimated to be more than 1,000 [2].

The propensity of researchers not to share patients' data may be resulting in an overprotection of privacy, which might not always be the preferred option by patients.

As a result, patients who want their data to be shared and accessed worldwide are creating their own platforms where they have the possibility to take direct responsibility for deciding between the benefits of sharing private information and the resulting risks to their privacy.

In patient led matchmaking databases, patients can access larger networks, disseminate information through other channels including social media and patient associations and provide detailed sets of phenotypic and genotypic data, often longitudinal in nature, even without expert mediation [55]. 
There is growing recognition that patients led research (PLR) can generate useful information for researchers, even though it has also been questioned for not being subject to ethical oversight and for methodological limitations including bias, self-selection, and problems with self-reporting of symptoms or phenotypic data $[71,72,73]$. Also the risk of engendering duplications and poor coordination of efforts with increased "false matches" may be a side effect of the multiplication of different genomics matchmaking databases [55].

Thus, patients and researchers must team up and involve each others in their research activities in order to build interoperable instruments and tools, and maximise the potential of matchmaking platforms while minimising informational risks for patients (including misuse of data, direct use of data for purposes not related with the aim of research, re-identifications, indirect diagnosis of family members and children).

In this "new model" of clinical diagnostics, families, patients, and scientists work jointly to find new patients, confirm or refute hypotheses and exchange clinical information [52].

Computational methods have the potential to overcome many problems associated with data sharing.

For instance, privacy-preserving record linking and applying FAIR principles 'at the source' further enables analysis across distributed data sources without moving and exposing sensitive data. Ontology mapping further provides sophisticated means to link patient-reported information and information provided by clinicians.

Besides, robust oversight mechanisms must be in place to maintain trust, including a governance system that involves patient associations and Research Ethics Committees (RECs) besides matchmaking platforms' data access committees, together with a secure system for accrediting and tracing the activity of researchers who are asking access to patients' genetic and phenotypic data [74].

If these conditions are met, it will be possible to increase the capacity for sharing far beyond what is permissible today, providing at the same time the possibility for patients to control the type of sharing to which they wish to grant trust.

\section{List of abbreviations}

Human Phenotype Ontology (HPO)

International Rare Disease Research consortium (IRDIRC)

Next generation Sequencing (NGS) 
Rare Diseases (RDs)

Syndromes Without A Name (SWAN)

Undiagnosed Disease Program (UDP)

Variant of Unknown Significance (VUS)

Whole Exome Sequencing (WES)

\section{Declarations}

Ethics approval and consent to participate: This work does not involve human participants, human data or human tissue and consequently ethics approval and informed consent are not required

Consent for publication: Not required

Availability of data and material: not applicable

Competing interests: The authors declare that they have no competing interests

Funding: This work has been supported by the European Union Seventh Framework Programme (FP7/2007-2013) under grant agreements no. 305444 (RD-Connect), 305121 (Neuromics), and no. 305608 (EURenOmics) as well as the BioBanking and Molecular Resource Infrastructure of Sweden project, Biobanking and Biomolecular Resources Research Infrastructure (BBMRI)LPC.

\section{Authors' contributions:}

SG conceived and designed the work, made the first draft of the manuscript and coordinated contributions by other co-authors;

GF was involved in drafting the manuscript and revising it critically for important intellectual content;

CP was involved in drafting the manuscript and revising it critically for important intellectual content;

VBF was involved in drafting the manuscript and revising it critically for important intellectual content;

MR was involved in drafting the manuscript and revising it critically for important intellectual content;

MS was involved in drafting the manuscript and revising it critically for important intellectual content;

DT was involved in drafting the manuscript and revising it critically for important intellectual content;

DM was involved in drafting the manuscript and revising it critically for important intellectual content;

Each author has given final approval of the version to be published. Each author has participated sufficiently in the work to take public responsibility for appropriate portions of the content; and agreed to 
be accountable for all aspects of the work in ensuring that questions related to the accuracy or integrity of any part of the work are appropriately investigated and resolved.

\section{Acknowledgements:}

This work has been supported by the European Union Seventh Framework Programme (FP7/2007-2013) under grant agreements no. 305444 (RD-Connect), 305121 (Neuromics), and no. 305608 (EURenOmics) as well as the BioBanking and Molecular Resource Infrastructure of Sweden project, Biobanking and Biomolecular Resources Research Infrastructure (BBMRI)LPC.

We thank the RD-Connect Patient Ethics Council and RD-Connect Patient Advisory Council (Jean Jacques Cassiman, Tracy Dudding, Muriel Gevrey, Emma Heslop, Joseph Irwin, Julian Isla, Sigurður Jóhannesson, Lydia Lemonnier, Chantal Loirat, Dorthe Lykke, Milan Macek, Caron Molster, Kay Parkinson, Odile Perrousseaux, Marita Pohlschmidt, Daniel Renault, Peter Reussner, Françoise Rouault, Balthasar Schaap, Inge Schwersenz, Chris Sotirelis, Oliver Timmis, Johannes van Delden, Marieke van Meel, Elizabeth Vroom and Urban Wiesing) and all RD-connect partners for their valuable inputs. 
References

1. Boycott KM, Vanstone MR, Bulman DE, MacKenzie AE. 2013. Rare-disease genetics in the era of next-generation sequencing: discovery to translation. Nat Rev Genet 14:681-691. doi:10.1038/nrg3555

2. Boycott KM, Rath A, Chong JX, et al. International cooperation to enable the diagnosis of all rare genetic diseases. Am J Hum Gen 2017;100;695-705. doi: https://doi.org/10.1016/j.ajhg.2017.04.003.

3. Hennekam RCM. Care for patients with ultra-rare disorders. Eur J Med Gen 2011;54(3): 220-4.

4. Genetic Alliance UK, the Wilhelm Foundation, EURORDIS (Rare Diseases Europe), Rare Voices Australia (RVA), the Canadian Organization for Rare Disorders (CORD), the Advocacy Service for Rare and Intractable Diseases' stakeholders in Japan (ASrid) and the National Organization for Rare Disorders (NORD). International Joint Recommendations to Address Specific Needs of Undiagnosed Rare Disease Patients. Available at: https://www.eurordis.org/publication/international-joint-recommendationsaddress-specific-needs-undiagnosed-rare-disease-patients (last access November 3, 2017)

5. Black N, Martineau F, Manacorda T. Diagnostic odyssey for rare diseases: exploration of potential indicators. 2015, Policy Innovation Research Unit. Available at:

http://www.piru.ac.uk/assets/files/Rare\%20diseases\%20Final\%20report.pdf (last access May 20, 2018).

6. Sox HC, Blatt MA, Higgins MC, et al. Medical decision making. Boston: Butterworth, 1988.

7. Haendel M. The application of the Human Phenotype Ontology. Presentation at the II International Summer School, Rare disease and orphan drug registries, organized by the National Centre for Rare Diseases, Istituto Superiore di Sanità, Rome, September 19th, 2014.

8. EURORDIS. 2007. Survey of the delay in diagnosis for 8 rare diseases in Europe (EurordisCare2). Fact sheet EurordisCare2.

9. Zurynski Y, Deverell M, Dalkeith T, et al. Australian children living with rare diseases: experiences of diagnosis and perceived consequences of diagnostic delays. Orphanet J Rare Dis. 2017:12:68.

https://doi.org/10.1186/s13023-017-0622-4

10. Gahl WA, Tifft CJ, The NIH Undiagnosed Diseases Program: lessons learned, J Am Med Assoc. 2011;305:1904-1905.

11. Taruscio D, Groft SC, Cederroth H,et al. Undiagnosed Diseases Network International (UDNI): White paper for global actions to meet patient needs. Mol Genet Metab. 2015;116(4):223-5. doi:

10.1016/j.ymgme.2015.11.003.

12. Taruscio D, Floridia G, Salvatore M, Groft S, Gahl WA. Undiagnosed diseases: International efforts to tackle rare and common diseases lacking a diagnosis. Posada de la Paz M, Groft S (Eds): Rare Diseases Epidemiology: Update and Overview, Adv Exp Med Biol. 2017;1031.

13. Gahl WA, Markello TC, Toro C, et al. The NIH Undiagnosed Diseases Program: Insights into Rare Diseases. Gen med. 2012;14(1):51-59. doi:10.1038/gim.0b013e318232a005.

14. Beaulieu CL, Majewski J, Schwartzentruber J, et al. FORGE Canada Consortium: Outcomes of a 2Year National Rare-Disease Gene-Discovery Project. Am J Hum Gen. 2014; 94(6):809-817. https://doi.org/10.1016/j.ajhg.2014.05.003.

15. Johnston L, Thompson R, Turner C, et al. The impact of integrated omics technologies for patients with rare diseases. Expert Opinion on Orphan Drugs. 2014;2(11):1211-9.

http://dx.doi.org/10.1517/21678707.2014.974554

16. The Global Alliance for Genomics and Health. A federated ecosystem for sharing genomic, clinical data. Science. 2016.352(6291):1278-1280. doi: 10.1126/science.aaf6162

17. http://www.irdirc.org/rare-diseases-research/current-results-of-research/

18. http://www.irdirc.org/irdirc-goals-2017-2027-new-rare-disease-research-goals-for-the-nextdecade/

19. Jameson JL, Longo DL. Precision Medicine -Personalized, Problematic, and Promising. New Engl J Med. 2015;372(23):2229-34. DOI: 10.1056/NEJMsb1503104.

20. Foo JN, Liu JJ, Tan EK. Whole-genome and whole-exome sequencing in neurological diseases. Nat Rev Neurol. 2012;8:508-517. doi:10.1038/nrneurol.2012.148 
21. Renkema KY, Stokman MF, Giles RH, Knoers NVAM. Next-generation sequencing for research and diagnostics in kidney disease. Nat Rev Nephrol. 2014;10: 433-444. doi:10.1038/nrneph.2014.95

22. Hardwick SA, Deveson IW, Mercer TR. Reference standards for next-generation sequencing. Nat rev Gen. 2017; doi:10.1038/nrg.2017.44

23. Baynam $G$, Walters $M$, Claes $P$, et al. Phenotyping: Targeting genotype's rich cousin for diagnosis. Journal of Paediatrics and Child Health. 2014;1-6. doi: 10.1111/jpc.12705

24. Hennekam RCM,Biesecker LG. Next-generation sequencing demands next-generation phenotyping; Hum Mutat. 2012; 33 (5): 884-886. DOI: 10.1002/humu.22048

25. Robinson PN. Deep phenotyping for precision medicine. Hum Mutat. 2012 May;33(5):777-80. doi: 10.1002/humu.22080.

26. Committee on the Framework for Developing a New Taxonomy of Disease. Toward Precision Medicine: Building a Knowledge Network for Biomedical Research and a New Taxonomy of Disease. 2011, National Academy of Sciences. Bookshelf ID: NBK92146

27. Lopez Martin E; Alonso V; Taruscio D; Rubinstein YR; Posada M. A mapping study of the biomedical ontologies in the RD-Connect project framework. 2014: figshare.

https://doi.org/10.6084/m9.figshare.939391.v1

28. Roos M, López Martin E, Wilkinson MD. Preparing data at the source to foster interoperability across rare disease resources. Adv Exp Med Biol 2017;1031:165-179. doi: 10.1007/978-3-319-67144-4_9.

29. Köhler S, Doelken SC, Mungall CJ, et al. The Human Phenotype Ontology project: linking molecular biology and disease through phenotype data. Nucleic Acids Res. 2014 Jan;42(Database issue):D966-74. doi: 10.1093/nar/gkt1026.

30. Köhler S, Vasilevsky NA, Engelstad M, et al. The Human Phenotype Ontology in 2017. Nucleic Acids Res. 2017;45(D1):D865-D876. doi: 10.1093/nar/gkw1039.

31. Girdea M, Dumitriu S, Fiume M, Bowdin S, Boycott KM, Chénier S, et al. PhenoTips: patient phenotyping software for clinical and research use. Hum Mutat. 2013 Aug;34(8):1057-65. doi: 10.1002/humu.22347. Epub 2013 May 24.

32. Köhler S, Schulz MH, Kravitz $\mathrm{P}$, et al. Clinical diagnostics in human genetics with semantic similarity searches in ontologies. Am J Hum Genet. 2009;85 (4): 457-64. https://doi.org/10.1016/j.ajhg.2009.09.003

33. Washington NL, Haendel MA, Köhler S, Lewis SE, Robinson P, Smedley D, Mungall CJ. 2014. How good is your phenotyping? Methods for quality assessment. In Proceedings of Phenotype Day 2014. Available at: http://phenoday2014.bio-lark.org/pdf/6.pdf

34. Gottlieb MM, Arenillas DJ, Maithripala S, Maurer ZD, TarailoGraovac M, Armstrong L, Patel M, Karnebeek C, Wasserman WW. 2015. GeneYenta: a phenotypeBased rare disease case matching tool based on online dating algorithms for the acceleration of exome interpretation. Hum Mutat. 36:432-438.

35. Bamshad MJ, Shendure JA, Valle D, et al. Centers for Mendelian Genomics. The Centers for Mendelian Genomics: a new large-scale initiative to identify the genes underlying rare Mendelian conditions. Am J Med Genet. 2012; A 158A:1523-1525.

36. Buske OJ, Girdea M, Dumitriu S, et al. PhenomeCentral: A Portal for Phenotypic and Genotypic Matchmaking of Patients with Rare Genetic Diseases. Hum Mutat. 2015;36(10):931-40. doi: 10.1002/humu.22851.

37. Chatzimichali EA, Brent S, Hutton B, et al. Facilitating Collaboration in Rare Genetic Disorders Through Effective Matchmaking in DECIPHER. Hum Mutat. 2015;36(10):941-9. doi: 10.1002/humu.22842.

38. Philippakis AA, Azzariti DR, Beltran S, et al. The Matchmaker Exchange: A Platform for Rare Disease Gene Discovery. Hum Mutat. 2015;36(10):915-21. doi: 10.1002/humu.22858.

39. Wilkinson M, Dumontier M, Aalbersberg IJ, et al. The FAIR Guiding Principles for scientific data management and stewardship. Scientific Data. 2016;3:160018. doi:10.1038/sdata.2016.18

40. No authors listed. Data models to GO-FAIR. Nature Genetics. 2017;49:971. doi:10.1038/ng.3910

41. Sawyer SL, Hartley T, Dyment DA, et al. Utility of whole-exome sequencing for those near the end of the diagnostic odyssey: time to address gaps in care. Clin Genet. 2016;89(3):275-84. doi: 10.1111/cge.12654. 
42. Fogel BL, Lee H, Strom SP, Deignan JL, Nelson SF. Clinical exome sequencing in neurogenetic and neuropsychiatric disorders. Ann N Y Acad Sci. 2016; 1366(1): 49-60. doi: 10.1111/nyas.12850

43. Walsh $\mathrm{M}$, Bell KM, Chong $B$, et al. Diagnostic and cost utility of whole exome sequencing in peripheral neuropathy. Ann Clin Transl Neurol. 2017, 4: 318-325. doi:10.1002/acn3.409

44. Monroe GR, Frederix GW, Savelberg SM, et al. Effectiveness of whole-exome sequencing and costs of the traditional diagnostic trajectory in children with intellectual disability. Genet Med. 2016 Sep;18(9):949-56. doi: 10.1038/gim.2015.200.

45. Harris E, Topf A, Barresi R, et al. Exome sequences versus sequential gene testing in the UK highly specialised Service for Limb Girdle Muscular Dystrophy. Orphanet J Rare Dis. 2017 Sep 6;12(1):151. doi: 10.1186/s13023-017-0699-9.

46. Stark Z, Schofield D, Alam K, et al. Prospective comparison of the cost-effectiveness of clinical whole-exome sequencing with that of usual care overwhelmingly supports early use and reimbursement. Genet Med. 2017 Aug;19(8):867-874. doi: 10.1038/gim.2016.221.

47. https://www.undiagnosed.org.uk/news-events/news/introducing-swan-europe

48. Tifft CJ, Adams DR. The National Institutes of Health undiagnosed diseases program. Curr Opin Pediatr. 2014;26(6):626-33

49. Gahl WA, Wise AL, Ashley EA. The Undiagnosed Diseases Network of the National Institutes of Health: a National extension. JAMA. 2015;314(17):1797-1798. doi:10.1001/jama.2015.12249

50. Baynam G, Broley S, Bauskis A, et al. Initiating an undiagnosed diseases program in the Western Australian public health system. Orphanet J Rare Dis. 2017;12:83. doi:10.1186/s13023-017-0619-z.

51. Drolet BC, Brower JP. The Undiagnosed Diseases Program. JAMA. 2016;315(17):1903-1904. doi:10.1001/jama.2016.0443

52. Might $M$, Wilsey $M$. The shifting model in clinical diagnostics: how next-generation sequencing and families are altering the way rare diseases are discovered, studied, and treated. Genet Med. 2014;16(10):736-7. doi: 10.1038/gim.2014.23.

53. Baldo C, Casareto L, Renieri A, et al. The alliance between genetic biobanks and patient organisations: the experience of the Telethon network of genetic biobanks. Orphanet J Rare Dis. 2016.

11:142. DOI 10.1186/s13023-016-0527-7

54. Graham CE, Molster C, Baynam GS, et al. Current trends in biobanking for rare diseases: a review. Journal of Biorepository Science for Applied Medicine. 2014,2:49-61. DOI

https://doi.org/10.2147/BSAM.S46707

55. Lambertson KF, Damiani SA, Might M, Shelton R, Terry SF. Participant-driven matchmaking in the genomic era. Hum Mutat. 2015 Oct;36(10):965-73. doi: 10.1002/humu.22852.

56. Salmon P. Conflict, collusion or collaboration in consultations about medically unexplained symptoms: The need for a curriculum of medical explanation. Patient Education and Counseling. 2007;67(3):246-54. doi:10.1016/j.pec.2007.03.008

57. Helman CG. Disease versus illness in general practice. J R Coll Gen Pract. 1981;31:548-52.

58. Vasilevsky NA, Foster ED, Engelstad ME, et al. Plain-language medical vocabulary for precision diagnosis. Nat genetics 2018;50:474-76. doi:10.1038/s41588-018-0096-x

59. Mascalzoni D, Dove E, Rubinstein Y, et al. International Charter of principles for sharing biospecimens and data. Eur J Hum Genet. 2015;23(6):721-8. doi: 10.1038/ejhg.2014.197.

60. Mascalzoni D, Paradiso A, Hansson MG. Rare disease research: Breaking the privacy barrier. Applied \& Translational Genomics. 2014. 3(2): p. 23-29. https://doi.org/10.1016/j.atg.2014.04.003

61. http://www.irdirc.org/activities/current-activities/privacy-preserving-record-linkage/

62. American Society of Human genetics. Genetic counseling. Am J Hum Genet. 1975 Mar;27(2):240-2.

63. Appelbaum PS, Parens E, Waldman CR, et al. Models of consent to return of incidental findings in genomic research. Hastings Center Report. 2014;44(4): p. 22-32. doi: 10.1002/hast.328.

64. Kaye J, Hurles M, Griffin $\mathrm{H}$, et al. Managing clinically significant findings in research: the UK10K example. Eur J Hum Genet. 2014. 22(9): p. 1100-1104. doi: 10.1038/ejhg.2013.290. 
65. Gliwa C, Berkman BE. Do researchers have an obligation to actively look for genetic incidental findings? American Journal of Bioethics. 2013. 13(2): p. 32-42. doi: 10.1080/15265161.2012.754062.

66. Kleiderman E, Knoppers BM, Fernandez CV, et al. Returning incidental findings from genetic research to children: views of parents of children affected by rare diseases. J Med Ethics. 2014;40(10):6916. doi: 10.1136/medethics-2013-101648.

67. Hallowell N, Hall A, Alberg C, Zimmern R. Revealing the results of whole-genome sequencing and whole-exome sequencing in research and clinical investigations: some ethical issues. J Med Ethics. 2015;41(4):317-21. doi: 10.1136/medethics-2013-101996.

68. Hayeems RZ, Miller FA, Bytautas JP, Li L. Does a duty of disclosure foster special treatment of genetic research participants? J Genet Couns. 2013. 22(5): p. 654-61.;

69. Green RC, Berg JS, Grody WW, et al. ACMG recommendations for reporting of incidental findings in clinical exome and genome sequencing. Genet Med. 2013;15(7):565-74. doi: 10.1038/gim.2013.73.

70. https://ec.europa.eu/health/sites/health/files/ern/docs/2017_brochure_en.pdf

71. Vayena E, Brownsword R, Edwards SJ, et al Research led by participants: a new social contract for a new kind of research Journal of Medical Ethics. 2015. doi: 10.1136/medethics-2015-102663.

72. Janssens AC, Kraft P. Research conducted using data obtained through online communities: ethical implications of methodological limitations. PLoS Med. 2012;9(10):e1001328. doi:

10.1371/journal.pmed.1001328.

73. Vayena E, Tasioulas J. Adapting Standards: Ethical Oversight of Participant-Led Health Research. PLoS Med. 2013 10(3): e1001402. https://doi.org/10.1371/journal.pmed.1001402.

74. Dyke SOM, Kirby E, Shabani M, Thorogood A, Kato K, Knoppers BM. Registered access: a "Triple-A" approach. Eur J Hum Gen. 2016;24(12):1676-1680. doi:10.1038/ejhg.2016.115. 
Box 1: Patient involvement in the search for a diagnosis

1) actively participate in the description of their phenotype and review the description that is made by professionals or contribute to the description of their phenotype which can be validated by clinicians or specifically trained experts (especially regarding the correct use of the terminology/recognised ontology); 2) be the ones to choose the level of visibility of their profile in matchmaking databases (e.g. private for clinicians, available to a closed community of researchers or public);

3) express their preferences regarding return of new findings, in particular regarding the level of significance a VUS should have in order to be considered relevant to them. 
Table 1: Values to be taken into account while collecting and sharing patients data in matchmaking databases

\begin{tabular}{|c|c|c|}
\hline Value at stake & Actions required & Interests to be balanced \\
\hline \multirow[t]{4}{*}{$\begin{array}{l}\text { Respect for patient } \\
\text { autonomy }\end{array}$} & $\begin{array}{l}\text { Involve patients in the diagnostic } \\
\text { process, provide adequate } \\
\text { information and alternatives }\end{array}$ & $\begin{array}{l}\text { Avoiding information overload } \\
\text { Respecting the right not to know }\end{array}$ \\
\hline & $\begin{array}{l}\text { Take into account patient self } \\
\text { reported phenotype (the patient } \\
\text { knows best the frequency, type and } \\
\text { intensity of his/her symptoms) }\end{array}$ & $\begin{array}{l}\text { Preserve scientific value, need to check } \\
\text { correctness of patient assertions }\end{array}$ \\
\hline & $\begin{array}{l}\text { Allow a role in the decisions } \\
\text { regarding the level of visibility and } \\
\text { "matchability" in matchmaking } \\
\text { platforms }\end{array}$ & $\begin{array}{l}\text { Promote the ability of other patients to } \\
\text { access information in order to be } \\
\text { diagnosed as well }\end{array}$ \\
\hline & $\begin{array}{l}\text { Allow a role in the decision } \\
\text { whether to be informed or not } \\
\text { about new findings as they emerge }\end{array}$ & $\begin{array}{l}\text { Evaluate scientific value of the } \\
\text { information } \\
\text { Evaluate the risk/benefit ratio (including } \\
\text { psychological harm) } \\
\text { Respect the right not to know }\end{array}$ \\
\hline \multirow[t]{2}{*}{ Beneficence } & $\begin{array}{l}\text { Maximise the potential of } \\
\text { diagnosing by potentiating } \\
\text { matchmaking platforms (produce } \\
\text { more data, make data } \\
\text { interoperable and accessible } \\
\text { worldwide in controlled settings) }\end{array}$ & $\begin{array}{l}\text { Protect patient confidentiality } \\
\text { Justify costs } \\
\text { Define criteria for deciding which } \\
\text { patients should be prioritised for NGS }\end{array}$ \\
\hline & $\begin{array}{l}\text { Offer patients adequate genetic } \\
\text { counselling to maximize benefits of } \\
\text { the results and avoid potential } \\
\text { harms deriving from } \\
\text { misinterpretations of genetic data }\end{array}$ & Justify costs \\
\hline Non-maleficence & Protect patient confidentiality & Promote potentially beneficial research \\
\hline
\end{tabular}




\begin{tabular}{|c|c|c|}
\hline & $\begin{array}{l}\text { Do not create anxiety } \\
\text { Do not raise wrong expectation or } \\
\text { false hope }\end{array}$ & \\
\hline Justice & $\begin{array}{l}\text { Balance access to healthcare } \\
\text { resources for all patients } \\
\text { Take into account the balance } \\
\text { cost/benefits with regard to: } \\
\text { - use of sequencing } \\
\text { techniques } \\
\text { - providing genetic } \\
\text { counselling to patients } \\
\text { managing and } \\
\text { communicating new } \\
\text { findings as they emerge }\end{array}$ & $\begin{array}{l}\text { Provide greater access for RD patients } \\
\text { that face more difficulties in getting } \\
\text { diagnosed (beneficience) } \\
\text { Do not provide information to patients } \\
\text { if genetic counselling is not available, } \\
\text { especially if the information may } \\
\text { generate anxiety (non } \\
\text { maleficence/autonomy) }\end{array}$ \\
\hline $\begin{array}{l}\text { Solidarity among } \\
\text { patients }\end{array}$ & $\begin{array}{l}\text { Make patients' genotype and } \\
\text { phenotype data available in order } \\
\text { to find matches }\end{array}$ & $\begin{array}{l}\text { Protect patient privacy and integrity } \\
\text { Respect patient decisions }\end{array}$ \\
\hline $\begin{array}{l}\text { Researchers reciprocity } \\
\text { with patients }\end{array}$ & $\begin{array}{l}\text { Make cases accessible in } \\
\text { accordance to the desire of } \\
\text { patients }\end{array}$ & $\begin{array}{l}\text { Preserve researchers capacity to stay } \\
\text { competitive and be able to publish } \\
\text { results }\end{array}$ \\
\hline
\end{tabular}

